PERIODONTOLOGY; METABOLIC MEDICINE

\section{The effect of periodontal therapy on uncontrolled type 2 diabetes mellitus in older subjects}

Promsudthi A, Pimapansri S et al. Oral Diseases 2005; 11: 293-298

Periodontal treatment improved periodontal health but did not affect glycaemic control.

Certain cohort studies have reported an improvement in type 2 diabetic metabolic control in response to periodontal treatment. However, the absence of a control group has led to the comment that behavioural factors were more likely to account for the change. In this study from Thailand, 60 type 2 diabetic patients aged 55-80 yrs, with HbA1c levels of 7.5-11.0\%, were randomised to non-surgical periodontal treatment (including adjunctive doxycycline) or control groups.

In the treatment group, 27 subjects completed the study, and in the control group, 25. Respective mean probing depths at baseline were 3.22 and $3.27 \mathrm{~mm}$, and at 3 months, $2.28(\mathrm{P}<0.05)$ and 3.28 $\mathrm{mm}$ (NS); respective mean clinical attachment levels changed from 4.03 to $3.58 \mathrm{~mm}(\mathrm{NS})$, and 4.10 to $4.34 \mathrm{~mm}(\mathrm{P}<0.05)$; respective mean BOP scores at baseline were 62 and 50\%, and at 3 months $24 \%(\mathrm{P}<0.05)$ and 50\%. However, respective mean $\mathrm{HbA1c}$ levels did not change significantly (8.98 to 8.78\% and 9.17 to $9.28 \%$ ). In the treatment group, 16 subjects had decreased $\mathrm{HbA1c}$, but in the control group, 14 also had decreased levels.

doi:10.1038/sj.bdj.4813218

\section{PERIODONTOLOGY; OBSTETRICS}

\section{Periodontal status and preterm low birth weight: a case control study}

Noack B, Klingenberg J et al. J Periodont Res 2005; 40: 339-345

Periodontal disease was not a risk factor for obstetric complications.

Some studies have suggested that periodontal disease in pregnancy may lead to preterm delivery and low birth weight (PTLB), but others have not confirmed this. This study in Germany compared periodontal status in 59 women having preterm contractions and 42 control women who had normal births. The former group was subsequently divided into two subgroups of 16 who actually had PTLB babies, and 43 who did not. Present or former smoking was a factor in only 10 subjects.

In all 3 groups, mean plaque and gingivitis scores, probing depths and attachment levels were similar. Regression analysis found no periodontitis-associated risk for PTLB babies. The authors discuss other studies showing similar results, and conclude that there is also no link between the conditions in this population.

\section{PROSTHODONTICS}

\section{A retrospective study of the prosthodontic management of patients with amelogenesis imperfecta}

Lindunger A, Smedberg J-I Int J Prosthodont 2005; 18: 189-194

Prosthodontic treatment improved both dental health and self-esteem.

There is a spectrum of treatment need in patients with amelogenesis imperfecta (AI). This Swedish study reports on the condition of 15 patients with AI aged 14-37 yrs (mean 23). Ten patients had hypoplastic type AI, and 5 had hypomineralised type. The patients had 403 teeth, of which 213 had restorations.

The restorations included 10 gold crowns, 121 metal-ceramic crowns, 28 ceramic crowns, 18 porcelain onlays or inlays, and 36 porcelain veneers. Only 1 crown was rated unsatisfactory according to CDA criteria for surface, colour, anatomical form and marginal integrity, and most restorations were graded excellent in all respects.

There had been 10 prosthetic complications of cementation or fracture, and in 1 patient, 11 crowns had been remade because of caries. Minor periodontal problems were noted. Patients had been very concerned about their teeth in adolescence, but treatment had greatly improved this. Seven patients felt the best age for treatment was between 10 and 15 yrs, and others, up to 25 yrs.

doi:10.1038/sj.bdj.4813220

CARIOLOGY; RADIOLOGY

Radiation caries - radiogenic destruction of dental collagen

Springer IN, Niehoff P et al. Oral Oncol 2005; 41; 723-728

This study suggests that irradiation does not have a direct effect on dental hard tissues, but may affect the pulp.

Ionizing radiation has well-known indirect effects on dental tissues by damaging salivary glands and reducing the protection of saliva against caries. Where salivary glands can be shielded, this effect can be reduced. This study of 80 extracted caries-free third molars from 36 patients aged 16-63 was to investigate whether radiation could have direct effects on dental tissues.

Half the teeth were exposed to 6.3 Gy per day for 5 days, and the rest were controls. Cementum was removed from roots, which were then separated from crowns. Pulps and dentine were pooled separately.

No difference in collagen structure was found in irradiated mineralised tissue, but collagen fragments were over 3 times as common in irradiated pulp. The authors suggest this effect may contribute to caries. 